\title{
Commentaries
}

\section{Genetics of inflammatory bowel disease: a puzzle with contradictions?}

Genetic factors have a well established role in the aetiology of inflammatory bowel disease (IBD). The mode of inheritance suggests a polygenic disease with penetrance of the genetic factors being strongly influenced by the lifestyle of an industrialised society. The development of linkage analysis has allowed the generation of important molecular clues to the location of putative disease genes.

The inheritance of genomic DNA can be traced by identification of highly polymorphic regions ("microsatellites"). Ideally, polymorphic regions should be used which are present in a high number of variations ("alleles") in the population (that is, are highly informative).

If a phenotype (for example, disease) is inherited, the use of linkage analysis assumes that it will be possible to trace the piece of DNA which contains the putative disease gene. In affected relative pairs of individuals (sharing the same phenotype), certain areas of the DNA which contain disease genes should therefore be coinherited more frequently in comparison with random distribution. In a systematic analysis ("genome scan"), a densely spaced set of microsatellites is used throughout the entire genome to identify the origin of DNA and follow them through the inheritance process. Statistical measures can be used to qualify the degree of over proportional sharing (of polymorphic alleles). This "LOD" score (which roughly corresponds to a logarithmised $p$ value) is usually displayed as a function of the genomic map (for example, fig 1 in Lesage and colleagues ${ }^{2}$ ). Linkage analysis typically leads to the description of a genomic area (often as large as one third of a chromosome), which most likely contains a disease gene.

It is obviously easier to detect disease genes in a monogenic disorder in which inheritance follows Mendelian rules. In many cases analysis of a few large families has led to the localisation of disease genes. In polygenic disorders, multiple disease genes are present and therefore linkage analysis has to be conducted using a population based approach. The method has to account for the fact that multiple disease genes may be present in varying combinations.

Siblings should share $50 \%$ of their DNA with each of their parents and also with each another. These DNA pieces should be randomly distributed, and sharing (in a sufficiently large sample) should not exceed $50 \%$ for any of the regions traced with microsatellites. Using siblings who share a disease will allow assessment of an over proportional amount of sharing which then can be used to describe a region in which disease genes are suspected.

In IBD a series of linkage studies have been conducted. The study by Hugot and colleagues ${ }^{3}$ described a region of chromosome 16 ("IBD1") which has been confirmed in a large number of subsequent studies. ${ }^{4-12}$ Satsangi and colleagues ${ }^{13}$ found a region on chromosome 12 ("IBD2") which was also confirmed in other studies. ${ }^{891415}$ We have described IBD 3 on chromosome $6^{9}{ }^{16}$ which was confirmed by Rioux and colleagues, ${ }^{17}$ and Brant and coworkers have suggested IBD4 (personal communication). In these stud- ies linkage was considered as verified based on the criteria of Kruglyak and Lander ${ }^{18}$ if the LOD score exceeded 3.6 in a single study and exceeded 2.0 in subsequent populations.

Using stochastic instruments for a population based analysis will always carry a significant amount of statistical uncertainty. Little is known of the theoretical specificity and power of linkage analysis. Therefore, we constructed a computer model of a six locus disease (three strong and three weak disease genes) and simulated transmission through random mating. ${ }^{19-21}$ Using a virtual population, repeat linkage studies can be simulated and different strategies for finding disease genes can be explored. From these studies it has become apparent that results from repeated linkage studies in the same population will result in LOD scores varying in a certain range. ${ }^{21}$ The power of linkage to detect disease genes in fairly high and increases with the number of sib pairs used (fig 1) ${ }^{21}$ However, using linkage for fine mapping of disease genes requires a high number of sib pairs, beyond technical and epidemiological feasibility.

In the study presented in this issue of Gut, Lesage and coworkes have conducted a linkage experiment limited to chromosome 12 (see page 787). ${ }^{2}$ They used families from all over Europe. In their study they could not replicate linkage on chromosome 12 which had been described previously by Satsangi and colleagues. ${ }^{13}$ Taking into account the results from mathematical modelling described above, the differences are not as surprising as they first appear: variance is expected in repetitive linkage studies which is greater in studies with small family numbers and in disease genes with a low impact on the susceptibility to disease development, respectively. Lesage and coworkers used 95 families, which is in the lower range of power in comparison with larger studies. ${ }^{2}$ All other studies which found linkage to chromosome 12 reported LOD scores much smaller than those described

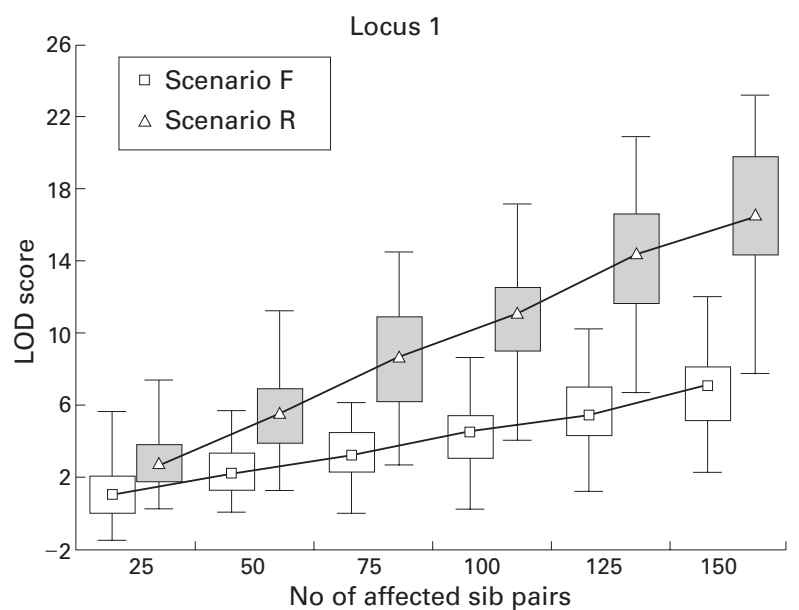

Figure 1 Variations in linkage results in repeated studies are dependent on the genetic impact of a locus and sample size. A population was simulated with a six locus disease, with three contributing loci of high frequency but low impact (scenario F) and three contributing loci with high impact but low frequency (scenario R). LOD score as a function of the number of analysed sib pairs is shown, as calculated by the SIPPAIR program in 60 replicate studies, which were conducted in sib pairs randomly drawn from the population. The analysis of locus 1 under both disease model scenarios ( $F=$ frequent, locus specific heritability $h 2=0.37$; $R=$ rare, $h 2=0.25$ ) is shown. Boxes denote median (interquartile range), and whiskers indicate minimum and maximum values (reprinted from Hampe and colleagues ${ }^{21}$ ). 
by Satsangi et al in the initial analysis. ${ }^{13}$ As concluded by Lesage and colleagues ${ }^{2}$ further studies are needed to determine if chromosome 12 contains a major disease gene or if the putative gene in this region has only a small influence on the risk of developing IBD.

A third possibility is that we are looking at a population specific difference. Although it appears unlikely that the population in the UK is substantially different from that in France or Belgium, only a repeat study in the UK could clarify this issue. Within our own linkage study ${ }^{9}$ much of the power for the weak linkage seen on chromosome 12 resulted from the sib pairs recruited from the UK which would support this theory (unpublished). A consortial analysis, which drew about $50 \mathrm{sib}$ pairs from each sample collected world wide, ${ }^{22}$ confirmed linkage to chromosome 12 although at a much lower significance level than that reported initially.

The work of Lesage and colleagues ${ }^{2}$ further indicates that linkage to chromosome 12 is not as powerful as initially thought. Identification of the first disease genes for IBD is still eagerly awaited. Although most likely in the distant future, only the definition of the causative mutations will allow a precise construction of a disease model and more exact predictions of the number and influence of additional genetic alterations.

Ist Department of Medicine,

S SCHREIBER

Christian-Albrechts-University,

Schittenhelmstrasse 12,

24105 Kiel, Germany

s.schreiber@mucosa.de

1 Hugot JP, Zouali H, Lesage S, et al. Etiology of the inflammatory bowel diseases. Int f Colorectal Dis 1999;14:2-9.

2 Lesage S, Zouali H, Colombel JF, et al. Genetic analyses of chromosome 12 loci in Crohn's disease. Gut 2000;47:787-91

3 Hugot JP, Laurent-Puig P, Gower-Rousseau C, et al. Mapping of a susceptibility locus for Crohn's disease on chromosome 16. Nature 1996;379:8213.
4 Ohmen JD, Yang HY, Yamamoto KK, et al. Susceptibility locus for inflammatory bowel disease on chromosome 16 has a role in Crohn's disease, but not in ulcerative colitis. Hum Mol Genet 1996,5:1679-83.

5 Parkes M, Satsangi J, Lathrop GM, et al. Susceptibility loci in inflammatory bowel disease. Lancet 1996;348:1588.

6 Cho JH, Fu Y, Kirshner BS, et al. Confirmation of a susceptibility locus, IBD 1, for Crohn's disease on chromosome 16. Inflamm Bowel Dis 1997;3:18690.

7 Cavanaugh JA, Callen DF, Wilson SR, et al. Analysis of Australian Crohn's disease pedigrees refines the localization for susceptibility to inflammatory bowel disease on chromosome 16. Ann Hum Genet 1998;62:291-8.

8 Curran M, Lau KF, Hampe J, et al. Genetic analysis of inflammatory bowel disease in a large European cohort supports linkage to chromosomes 12 and 16. Gastroenterology 1998;115:1-7.

9 Hampe J, Schreiber S, Shaw S, et al. A genomewide analysis provides evidence for novel linkages in inflammatory bowel disease in a large European cohort. Am f Hum Genet 1999;64:808-16.

10 Brant SR, Fu Y, Fields CT, et al. American families with Crohn's disease have strong evidence for linkage to chromosome 16 but not chromosome 12. Gastroenterology 1998;115:1056-61.

11 Mirza MM, Lee J, Teare D, et al. Evidence of linkage of the inflammatory bowel disease susceptibility locus on chromosome 16 (IBD1) to ulcerative bowel disease susceptibility locus on chr
colitis. F Med Genet 1998;35:218-21.

12 Annese V, Latiano A, Bovio P, et al. Genetic analysis in Italian families with inflammatory bowel disease supports linkage to the IBD1 locus-a GISC study. Eur F Hum Genet 1999;7:567-73.

13 Satsangi J, Parkes M, Louis E, et al. Two stage genome wide search in inflammatory bowel disease provides evidence for loci on chromosomes 3 , 7 and 12. Nat Genet 1996,14:199-202.

14 Duerr RH, Barnada M, Zhang L, et al. Linkage and association between inflammatory bowel disease and a locus on chromosome 12. Am f Hum Genet 1998;63:95-100.

15 Yang H, Ohmen JD, Ma R, et al. Linkage and association between Crohn's disease and a putative locus on chromosome 12. Gastroenterology 1998;114: A902.

16 Hampe J, Shaw SH, Saiz R, et al. Linkage of inflammatory bowel disease to human chromosome 6p. Am f Hum Genet 1999;65:1647-55.

17 Rioux JD, Silverberg MS, Daly MJ, et al. Genomewide search in Canadian families with inflammatory bowel disease reveals two novel susceptibility loci. Am F Hum Genet 2000;66:1863-70

18 Kruglyak L, Lander E. Genetic dissection of complex traits: Guidelines for interpreting and reporting linkage results. Nat Genet 1995;11:241-7.

19 Kruglyak L, Lander ES. High-resolution genetic mapping of complex traits. Am f Hum Genet 1995;56:1212-23.

20 Hampe J, Wienker T, Schreiber S, et al. POPSIM: A general population simulation program. Bioinformatics 1998;14:458-64.

21 Hampe J, Wienker T, Nürnberg P, et al. Mapping genes for inflammatory bowel disease: Considerations for study design in a complex trait. Hum Hered 2000;50:91-101.

22 Cavanaugh $\mathrm{J}$ for the IBD genetics consortium. The international IBD genetics consortium confirms linkage of Crohn's disease to a locus on chromosome 16 (IBD1). Gastroenterology 2000;118:A3862.

\section{Gastrin, growth, and colon neoplasia}

The ability of the gastric hormone gastrin to stimulate gut epithelial cell proliferation has been appreciated since the late 1960 s. ${ }^{1}$ However, aside from the special case of gastric carcinoid tumours arising from enterochromaffin-like cells, the contribution of gastrin to gastrointestinal neoplasia has been uncertain. Several developments now suggest a role for gastrin in both gastric and colorectal cancer. In the case of gastric cancer, recent evidence indicates a synergy between gastrin and Helicobacter infection in accelerating progression to atrophy and cancer. ${ }^{2}$ Different issues are involved in colorectal cancer. The important emerging concepts here are that (a) the gastrin gene is expressed in colorectal cancer cells, but (b) the main products of gene expression in these cells are not ligands for the gastrincholesystokinin receptor B (CCKB), although (c) they do act as colon growth factors. The paper by Smith and Watson $^{3}$ now shows that gastrin mRNA, detected by reverse transcription-polymerase chain reaction, is expressed in early stage polyps, that the main forms of gastrin detected by immunohistochemistry are biosynthetic precursor peptides, and that there is also parallel expression of the gastrin-CCKB receptor (see page 820).

It used to be thought that the only biologically active peptides generated from the gastrin gene were $\mathrm{COOH}$ terminally amidated peptides such as G34 and G17, and that carboxy terminal amidation was essential for the biological activity of members of the gastrin family. The amidated gastrins are generated from a precursor, progastrin, via biosynthetic intermediates, the Gly-gastrins (fig 1). Neither progastrin nor Gly-gastrins have a carboxy terminal amide group and therefore they do not have high affinity for the gastrin-CCKB receptor, which is the receptor mediating acid secretory responses to gastrin. It seems, however, that these peptides exhibit other biological properties, notably stimulation of colon cell growth. This effect has been found in both transgenic mice over expressing progastrin and Gly-gastrin, and in colon cancer cell lines. ${ }^{4-6}$ The precise receptors mediating the trophic actions of progastrin and Gly-gastrin remain uncertain. It is not clear if there is one (the putative gastrin-CCKC receptor) or several. It is conceivable that a modified version of the gastrin-CCKB receptor, perhaps generated by alternative mRNA splicing, mediates the effects of progastrin and Gly-gastrin; at present this idea is largely speculative. It is unlikely, however, that the progastrin and Gly-gastrin found by Smith and Watson in early stage polyps act at authentic gastrin-CCKB receptors.

The pattern of relatively high abundance of progastrin and Gly-gastrin compared with amidated gastrin that occurs in colonic neoplasms differs markedly from that in normal pyloric antral $G$ cells where the amidated gastrins predominate. There are several plausible explanations for this. There may, for example, be differences in the expression of enzymes required for each conversion step in 


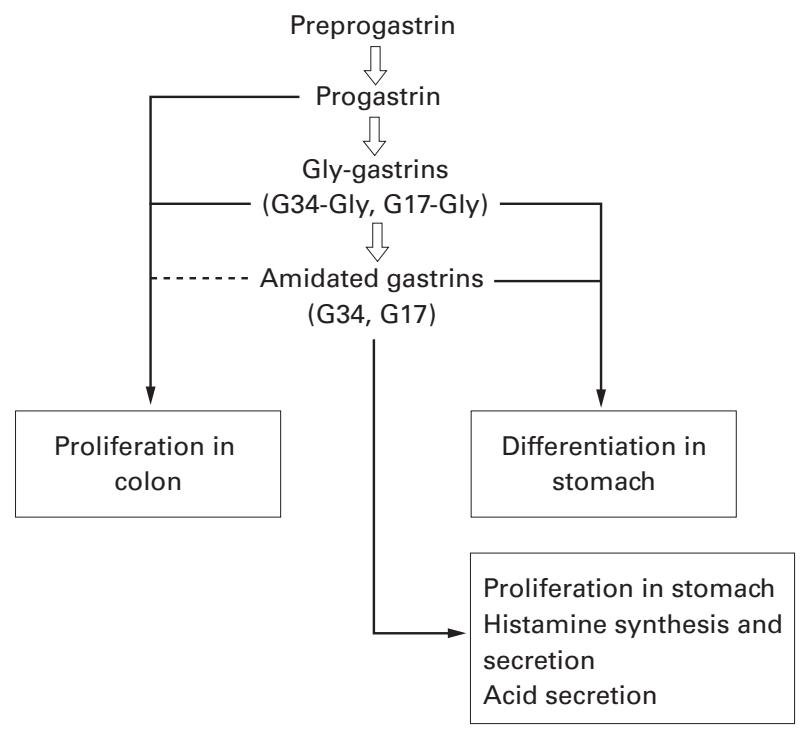

Figure 1 Schematic representation of the pathway of gastrin biosynthesis and the biological properties of the products. Open arrows show the conversion steps by which preprogastrin is converted to amidated gastrins. This sequence of events is completed in $G$ cells but in colorectal cancer cells the main products are progastrin and Gly-gastrin. Progastrin and

Gly-gastrins stimulate colon proliferation. Amidated gastrins play a minor role in control of colon proliferation but stimulate acid secretion and gastric proliferation via the gastrin-CCKB receptor (at which progastrin and Gly-gastrin have low affinity).

gastrin biosynthesis (fig 1). In normal G cells, the enzymes responsible for production of carboxy terminal amidated gastrins mostly act in vesicles of the so called regulated pathway of exocytosis ${ }^{7}$; but these vesicles are scarce or absent in polyp and adenoma cells. As a consequence, these cells are poorly equipped both to store hormonal peptides and to complete the full sequence of events giving rise to carboxy amidated gastrins. Instead, peptides mostly corresponding to progastrin and Gly-gastrin are likely to pass directly from the Golgi complex to the cell surface by what is known as the constitutive route of secretion.

What regulates gastrin gene expression in colon polyps and adenomas? Studies by Nakata et al have shown that oncogenic Ras stimulates the mitogen activated protein kinase pathway and so increases gastrin gene expression. ${ }^{8}$ Recently, Koh et al demonstrated that the $\beta$-catenin/T cell factor 4 pathway also stimulates gastrin gene expression. ${ }^{9}$ Acquisition of mutations that activate the latter pathway is likely to be an early event in the progression to colorectal cancer and could account for the increased gastrin gene expression found by Smith and Watson. Whether or not similar mechanisms account for upregulation of the gastrin-CCKB receptor is not known.

Recently, Singh et al reported that mice with elevated plasma progastrin exhibit increased aberrant crypt foci, adenomas, and adenocarcinomas after treatment with azoxymethane..$^{10}{ }^{11}$ Aberrant crypt foci are considered to be a marker for progression to colon cancer. Singh et al suggest progastrin is a cocarcinogen, that is, on its own it is not carcinogenic but it increases the pool of transformed cells and so exacerbates the oncogenic progression. Interestingly, mice with elevated plasma concentrations of amidated gastrin did not exhibit increased aberrant crypt foci, adenomas, or carcinomas in response to azoxymethane. The picture emerges then that mutations acquired early in the progression to colorectal cancer lead to increased local production of progastrin and Gly-gastrin that then act as auto- or paracrine growth factors expanding the number of transformed cells. This analysis leaves open the role of the gastrin-CCKB receptor, and further work will certainly be needed on this point. Whether or not these findings can be developed into novel therapeutic strategies will depend on characterisation of the receptor mechanisms mediating the effects of progastrin or Gly-gastrin on colon epithelial proliferation.

G J DOCKRAY

Physiological Laboratory,

University of Liverpool, Crown St,

PO Box 147, Liverpool L69 3BX, UK

g.j.dockray@liverpool.ac.uk

1 Johnson LR. Regulation of gastrointestinal mucosal growth. Physiol Rev 1988;68:456-502.

2 Wang TC, Dangler CA, Chen D, et al. Synergistic interaction between hypergastrinemia and Helicobacter infection in a mouse model of gastric cancer. Gastroenterology 2000;118:36-47.

3 Smith AM, Watson SA. Gastrin and gastrin receptor activation - an early event in the adenoma carcinoma sequence. Gut 2000;47:820-4.

4 Wang TC, Koh TJ, Varro A, et al. Processing and proliferative effects of human progastrin in transgenic mice. F Clin Invest 1996;98:1918-29.

5 Koh TJ, Dockray GJ, Varro A, et al. Overexpression of glycine-extended gastrin in transgenic mice results in increased colonic proliferation. $\mathcal{F}$ Clin Invest 1999;103:1119-26.

6 Hollande F, Imdahl A, Mantamadiotis T, et al. Glycine-extended gastrin acts as an autocrine growth factor in a nontransformed colon cell line. Gastroenterology 1997;113:1576-88.

7 Varro A, Henry J, Vaillant C, et al. Discrimination between temperature- and brefeldin A-sensitive steps in the sulfation, phosphorylation, and cleavage of progastrin and its derivatives. F Biol Chem 1994;269:20764-70.

8 Nakata H, Wang S-L, Chung DC, et al. Oncogenic ras induces gastrin gene Nakata H, Wang S-L, Chung DC, et al. Oncogenic ras induces g.
expression in colon cancer. Gastroenterology 1998;115:1144-53.

9 Koh TJ, Bulitta CJ, Fleming JV, et al. Gastrin is a downstream target of the $\beta$-cantenin/TCF-4 signaling pathway which mediates growth in the $\mathrm{APC}^{\mathrm{min}-/+}$ mouse model of intestinal polyposis. $\mathcal{F}$ Clin Invest 2000;106:5339 .

10 Singh P, Velasco M, Given R, et al. Progastrin expression predisposes mice to development of colon carcinomas and adenomas in response to AOM. Gastroenterology 2000;119:162-71.

11 Singh P, Velasco M, Given R, et al. Mice overexpressing progastrin are predisposed for developing aberrant colonic crypt foci in response to AOM. Am $\mathcal{F}$ Physiol Gastrointest Liver Physiol 2000;278:G390-9.

\section{Alcohol and retinoid metabolism}

The complex interactions between the metabolism of retinoids and ethanol have been reported for a long time. Clinically, chronic ethanol consumption leads to vitamin A deficiency but also to enhanced toxicity of vitamin A and beta-carotene when supplemented. Changes in retinol metabolism due to alcohol may have a pathophysiological impact in both alcoholic liver disease and alcohol associated cancer as retinoic acid, the most active form of vitamin A, is an important regulator of normal epithelial cell growth, function, and differentiation. Under normal conditions, ingested retinol is metabolised to retinaldehyde via cytosolic alcohol dehydrogenase $(\mathrm{ADH})$, microsomal retinol dehydrogenase (three types), and several types of cytosolic retinol dehydrogenases, and retinaldehyde is further oxidised to retinoic acid via aldehyde dehydrogenase (ALDH). Retinoic acid binds to retinoic acid receptors (RAR), initiating intracellular signal transduction leading to a cascade of events and finally to a decrease in cell regeneration. The main molecular action of retinoic acid involves either transactivation through direct binding to retinoic acid response elements (RARE) in target gene promoters, thereby transcriptionally activating a series of genes with distinct antiproliferative activity, or transrepression of activator protein (AP-1) and regulation of apoptosis. It is not surprising that this complex interaction between ethanol and metabolism of retinoids occurs as 
both substrates share common pathways, namely (a) ADH, (b) ALDH, and (c) cytochrome P4502E1 (CYP2E1).

It has been shown that chronic ethanol consumption decreases hepatic retinol and retinoic acid concentrations due to various mechanisms including, increased mobilisation of retinyl esters to extrahepatic tissues and enhanced hepatic metabolism of retinol and retinoic acid to polar metabolites, predominantly via induced CYP2E $1 .{ }^{12}$ These metabolites include 18-OH-retinoic acid, 4-OXO-retinoic acid, and some unidentified metabolites, possibly with fibrogenic and toxic properties. Decreased hepatic retinoic acid concentrations are associated with functional downregulation of RAR, enhanced expression of $\mathrm{AP}-1$ gene (c-jun and c-fos), and increased hepatic cell regeneration, all of which return to normal following retinoic acid supplementation. ${ }^{23}$ In contrast, retinol concentrations in extrahepatic tissues such as the oesophageal and colonic mucosa were found to be increased rather than decreased following chronic ethanol consumption. ${ }^{4}$ This was also confirmed in alcoholics with oropharyngeal cancer where normal retinol concentrations were found in normal oral mucosa adjacent to cancerous tissue. ${ }^{5}$ It was believed that one mechanism for this observation was increased mobilisation of retinyl esters from the liver to the oral mucosa.

In this issue of Gut, Parlesak and colleagues ${ }^{6}$ demonstrate another explanation for the lack of loss of retinol in gastrointestinal mucosa of alcoholics (see page 825 ). These authors showed inhibition of retinol oxidation by ethanol concentrations frequently observed after social drinking in an in vitro study using cytosol from rat liver and intestine. This effect was due to inhibition of $\mathrm{ADH}$. As this inhibition was found not only with low ethanol concentrations (8.6 $\mathrm{mM}$ ) but also increasingly with higher ethanol concentrations (17 mM, $34 \mathrm{mM}$ ), a low $\mathrm{K}_{\mathrm{m}} \mathrm{ADH}$ (rat ADH3 corresponding to class $\mathrm{I} \mathrm{ADH}$ ) and an $\mathrm{ADH}$ with higher $\mathrm{K}_{\mathrm{m}}$ (ADH 2 corresponding to class III ADH or ADH1 corresponding to class IV $\mathrm{ADH}$, not present in the rat liver and colon, but in the rat rectum ${ }^{7}$ ) seem to be involved. This inhibition of retinol metabolism by ethanol seems especially relevant in the colon. This may explain the accumulation of retinol in this tissue which may lead to a reduction in retinoic acid levels; however, this needs to be proved. In contrast with the colon, both retinol and retinoic acid concentrations were found to be significantly decreased in rat liver following chronic ethanol ingestion. ${ }^{2}$ Thus this finding cannot be explained by inhibition of retinol oxidation by ethanol. Indeed, at least in the liver, a variety of other pathways for oxidation of retinol are involved, as mentioned above.

In humans it has been shown that retinol is a physiological substrate for $\mathrm{ADH} 3$ (class $\mathrm{I} \mathrm{ADH}$ ) in the gastrointestinal mucosa. ADH3 has a low $K_{m}$ for ethanol (1-2 $\mathrm{mM}$ ) and is the only class I ADH gene that contains an RARE in the promoter region. It has been suggested that retinoic acid activation of ADH constitutes a positive feedback loop regulating retinoic acid synthesis. ${ }^{8}$ Ethanol was found to be a competitive inhibitor of retinol for class I $\mathrm{ADH}$, but also for classes II and IV ADH. ${ }^{9}{ }^{10}$ Indeed, it has been shown that class IV ADH (only present in the mucosa of the upper gastrointestinal tract) has a low $\mathrm{K}_{\mathrm{m}}$ for all-trans-retinol of 15-60 $\mu \mathrm{M}$ and has the highest catalytic efficiency of $3800-4500 \mathrm{mmol} / \mathrm{min} .{ }^{10}{ }^{11}$ In vitro studies using class IV ADH enzyme preparations have shown strong inhibition of metabolism of all-trans-retinol and 9-cis-retinol by ethanol with a $\mathrm{K}_{\mathrm{i}}$ of $6-10 \mathrm{mM} .{ }^{10}$ Oxidation of retinol to retinaldehyde is probably the rate limiting step in the generation of retinoic acid. However, recently it was shown in the rat oesophagus that in addition to ethanol, acetaldehyde also inhibits generation of retinoic acid, possibly by inhibition at the retinaldehyde level. ${ }^{12}$
The data of Parlesak et al extended these in vitro experiments with isolated $\mathrm{ADH}$ by using rat cytosol from liver and colonic mucosa. An important next step would be to measure retinoic acid in colonic mucosa following chronic ethanol consumption and, as there are differences in $\mathrm{ADH}$ patterns between rats and humans, to also measure retinoic acid in the mucosa of alcoholics.

It is interesting that class IV in contrast with class I ADH is not expressed in human colorectal mucosa. However, it was found recently that in a number of biopsies from colorectal polyps of alcoholics, class IV $\mathrm{ADH}$ was expressed. ${ }^{13}$ One explanation for such de novo expression of class IV $\mathrm{ADH}$ could be retinoic acid deficiency in a critical premalignant condition to guarantee increased generation of retinoic acid.

Chronic alcohol consumption is associated with an increased risk of both hepatic and colorectal cancer. Whereas for the liver, cirrhosis is possibly the most important precondition for cancer development, mechanisms for colorectal carcinogenesis are complex and less clear. One important morphological feature of chronic ethanol consumption in rats and humans is colorectal cellular hyperproliferation associated with extension of the proliferative compartment of the crypt towards the lumen, a condition associated with increased cancer risk. ${ }^{14}$ This early event in colorectal carcinogenesis was thought to be related to acetaldehyde induced cell injury as acetaldehyde (produced during mucosal and bacterial ethanol metabolism) and crypt cell production rate showed a significant positive correlation. ${ }^{14}$ However, this important alteration in cell cycle behaviour, which was also observed in the upper alimentary tract, could also be due to retinoic acid deficiency, and acetaldehyde may contribute by preventing its generation, as discussed above.

In summary, the paper by Parlesak et al shows the importance of ethanol in the inhibition of retinol metabolism in the liver and colon. However, it raises more questions than it answers. One major question arising from these data is whether decreased retinoic acid concentrations can be found in extrahepatic tissues, especially in the colorectal mucosa, following chronic ethanol ingestion of rodents, and even more important in alcoholic. If so, a new mechanism for alcohol associated carcinogenesis has to be considered.

H K SEITZ

Department of Medicine, Salem Medical Centre

and Laboratory of Alcohol Research, Liver Disease and Nutrition,

Heidelberg, Germany

1 Leo MA, Lieber CS. Alcohol, vitamin A, and beta-carotene: adverse interactions including hepatotoxicity and carcinogenesis. Am f Clin Nutr 1999;69:1071-85.

2 Wang XD, Liu C, Chung JY, et al. Chronic alcohol intake reduces retinoic acid concentrations and enhances AP-1 (c-jun and c-fos) expression in rat liver. Hepatology 1998;28:744-50.

3 Wang XD, Chung JY, Liu C, et al. Suppression of alcohol induced hepatocellular proliferation by all-trans retinoic acid. Alcoholism Clin Exp Res 2000;24(suppl): 188A.

4 Mobarhan S, Seitz HK, Russell RM, et al. Age-related effects of chronic ethanol intake on vitamin A status in Fisher 344 rats. $\mathcal{F}$ Nutr 1991;121:51017.

5 Leo MA, Seitz HK, Maier H, et al. Carotinoid, retinoid and vitamin E status of the oropharyngeal mucosa in the alcoholic. Alcohol Alcohol 1995;30:16370

6 Parlesak A, Menzl I, Feuchter A, et al. Inhibition of retinol oxidation by ethanol in the rat liver and colon. Gut 2000;47:825-31.

7 Boleda D, Julia P, Moreno A, et al. Role of extrahepatic alcohol dehydrogenase in rat ethanol metabolism. Arch Biochem Biophys 1989;274:74-81.

8 Duester G, Shean ML, McBride MS, et al. Retinoic response element in the human alcohol dehydrogenase gene $\mathrm{ADH} 3$ : implication for regulation of retinoic acid synthesis. Mol Cell Biol 1991;11:1638-45.

9 Han CL, Liao CS, Wu CW, et al. Contribution to first pass metabolism of ethanol and inhibition by ethanol for retinol oxidation in human alcohol dehydrogenase family: implications for etiology of fetal alcohol syndrome and alcohol related diseases. Eur F Biochem 1998;254:25-31.

10 Allali-Hassani A, Peralba JM, Martras S, et al. Retinoids, omega-hydroxy fatty acids and cytotoxic aldehydes as physiological substrates, and H2-receptor antagonists as pharmacological inhibitors of human class IV H2-receptor antagonists as pharmacological inhibit
alcohol dehydrogenase. FEBS Lett 1998;426:362-6.

11 Kedishvili NY, Gough WH, Davis WI, et al. Effect of cellular retinol binding protein on retinol oxidation by human class IV retinol/alcohol dehydroge- 
nase and inhibition by ethanol. Biochem Biophys Res Commun 1998;249: 191-6.

12 Shiraishi $\mathrm{H}$, Yokoyama $\mathrm{H}$, Matsumoto $\mathrm{M}$, et al. Characterization of oxidation pathway from retinal to retinoic acid in esophageal mucosa. Alcohol Clin Exp Res 2000;24(suppl):168A.
13 Seitz HK. Alcohol and colorectal cancer. Alcohol Clin Exp Res 2000;

24(suppl):188A.
14 Simanowski UA, Suter PM, Russell RM, et al. Enhancement of ethanol induced rectal hyperproliferation with age in F344 rats. Gut 1994;35: $1102-6$

\section{Web extra}

A new website has been developed to help gastroenterologists calculate CDAI (Crohn's Disease Activity Index) and PCDAI (Paediatric Crohn's Disease Activity Index).

The user registers his/her patient under a name/code and registers the sex, age, and weight of the patient, and is then asked to fill in raw data. The website will then calculate CDAI (and for patients below 19 years, PCDAI). The site enables the user to store indices over time, and the disease activity of patients will also be presented as a diagram. The data are stored on the Örebro Medical Centre Hospital for one year. You can visit the website at:

\section{http://www.orebroll.se/rso/barn/crohn/Home_Eng.asp}

Users must first register; this is done by entering a user name and password, which the user chooses (user names and passwords are not distributed by Dr Ludvigsson).

For further information: Dr Jonas Ludvigsson,

Scientific Coordinator,

Department of Paediatrics,

Örebro Medical Centre Hospital,

70185 Örebro,

Sweden

jonasludvigsson@yahoo.com www.geocities.com/jonasludvigsson 